\title{
Uso de interpoladores espaciais na estratificação estatística de Pinus taeda
}

\author{
Use of spatial interpolators for statistic stratification of Pinus taeda
}

\author{
Diego Fernando Zech ${ }^{1}$, Julio Cesar Refosco ${ }^{2}$, \\ Lia Toiosima Yoshizumi ${ }^{3}$ e Rafael Olivares de Salles Abreu ${ }^{4}$
}

\begin{abstract}
Resumo
Este estudo buscou verificar a eficiência dos interpoladores espaciais ponderação do inverso da fistância e krigagem ordinária sobre três variáveis dendrométricas: volume, área basal e altura dominante, na estratificação de um povoamento de Pinus taeda L. com idade de 19 anos. Os resultados foram comparados aos gerados por amostragem casual simples e amostragem casual estratificada. Para a krigagem, a interpolação da área basal foi a mais eficiente, reduzindo $73,42 \%$ da variância do volume e $28,59 \%$ do erro de amostragem do inventário. Para a ponderação pelo inverso da distância, a interpolação mais eficiente foi sobre o volume, a qual reduziu $79,21 \%$ da variância e $54,23 \%$ do erro de amostragem do inventário. Conclui-se que volume, área basal e altura dominante, apresentaram moderada dependência espacial. Além disso, ambos os interpoladores analisados se mostraram eficientes na estratificação florestal de povoamentos de Pinus taeda.
\end{abstract}

Palavras-chave: krigagem; geoestatística; inventário florestal.

\begin{abstract}
This study aimed to verify the efficiency of two spatial interpolators: Inverse distance weight and ordinary kriging, over three dendrometric variables: volume, basal area and dominant height in the stratification of a Pinus taeda L. plot at age 19 years. Results were compared to those resulted from simple casual sampling and stratified casual sampling. For kriging, the most efficient interpolation was obtained using basal area, which reduced $73.42 \%$ of the volume variance and $28,59 \%$ of sampling error. For Inverse distance weight, the most efficient interpolation was obtained using volume, which reduced $79.21 \%$ of variance and reduced $54.23 \%$ of sampling error. The conclusion is that the use of interpolation for Pinus taeda is efficient for the stratification. Moreover both used interpolators were efficient in the forest stratification of Pinus taeda populations.
\end{abstract}

Keywords: Kriging; geostatistic; forest inventory.

\section{INTRODUÇÃO}

O planejamento e a administração de recursos florestais exigem um alto nível de informações, o que traz a necessidade de aprimoramento contínuo das estimativas dos inventários florestais (MELLO et al., 2005a; PELISSARI et al., 2014). Isto, contudo, não ocorre gratuitamente. Ao ganho de qualidade nos inventários corresponde maior gasto de tempo e de recursos financeiros e depende de diversos fatores, como, por exemplo, da intensidade amostral (LUNDGREN; SILVA; FERREIRA, 2015).

A intensidade amostral é um parâmetro do inventário ligado diretamente com a precisão das estimativas. Este parâmetro varia com o número de unidades amostrais, com o controle da variância e, portanto, com o custo do inventário. (SANQUETTA et al., 2006). Por isso, o controle da variância em inventários florestais é o ponto central para uma boa relação entre custo e representatividade (KANEGAE JÚNIOR et al., 2006).

Mas, como controlar a variância? A estratificação florestal é uma das formas encontrada para controlar a variância dentro da floresta. Este procedimento de amostragem consiste, basicamente, em dividir a população em subpopulações mais homogêneas e, portanto, com menor variância, chama-

\footnotetext{
${ }^{1}$ Mestre em Engenharia Florestal. FURB - Universidade Regional de Blumenau. Rua José Dalmolinni, 60 - 89030-000 Guaramirim, SC, Brasil. E-mail: diegozech@gmail.com.

2Professor do Programa de Pós Graduação em Engenharia Florestal. FURB - Universidade Regional de Blumenau. Rua São Paulo, 3250 - 89030-000 - Blumenau, SC, Brasil. E-mail: refusco@fur.br.

${ }^{3}$ Engenharia Florestal. UFPR - Universidade Federal do Paraná. Av. Prefeito Lothario Meissner - Jardim Botânico - 80210170 - Curitiba, PR, Brasil. E-mail: liaty@klabin.com.br.

${ }^{4}$ Graduando em Engenharia Florestal. UFLA - Universidade Federal de Lavras. Campus Universitário - Caixa-postal: 3037 37200-000 - Lavras, MG, Brasil. E-mail: roabreu@klabin.com.br.
} 
dos estratos. Via de regra os procedimentos de amostragem que se baseiam na estratificação são mais precisos do que aqueles baseados em processos puramente aleatórios (MELLO; SCOLFORO, 2000), tendo em conta que esta precisão está relacionada com a variabilidade da população a ser amostrada.

O estabelecimento dos estratos, contudo, deve ser realizada por meio de um processo criterioso. Em geral, os estratos são definidos com base em informações cadastrais da população, mas a estratificação mais eficiente, porém, é aquela baseada na própria característica de interesse (PÉLLICO NETTO; BRENA, 1997). Neste sentido, o uso da interpolação espacial na estratificação tem chamado atenção. Estudos como os de KANEGAE et al. (2006), Assis et al. (2009) e Guedes et al. (2012) encontraram expressiva redução do erro de amostragem com o uso de interpoladores espaciais em plantios jovens de Eucalyptus sp.

Existem, entretanto, diversos interpoladores espaciais, cada um com características mais ou menos adequadas para uso na estratificação em inventários florestais. A princípio os interpoladores espaciais podem ser classificados em estatísticos e determinísticos. O estatístico é também conhecido como krigagem e, dentre os determinísticos, destaca-se aqui o interpolador Ponderação do Inverso da distância, também conhecido pelo seu nome em inglês: Inverse Distance Weighting (IDW). Uma das principais diferenças entre eles é que a condição impositiva para o uso da krigagem é a existência de correlação espacial dos dados (YAMAMOTO; LANDIM, 2013). Por outro lado, a interpolação para cada espécie, cada idade e parâmetro dendrométrico considerado pode também apresentar particularidades quanto ao uso de interpoladores espaciais.

Assim, considerando este contexto, os objetivos desse estudo foram: i) avaliar o uso dos interpoladores espaciais IDW e krigagem, na estratificação florestal de um povoamento de Pinus taeda, utilizando diferentes parâmetros dendrométricos; ii) comparar a variância da média do volume e o erro de amostragem gerados por estimadores da amostragem casual simples e da amostragem casual estratificada do inventário florestal com os estratos baseados nos mapas gerados pelos diferentes interpoladores.

\section{MATERIAL E MÉTODOS}

\section{Área de estudos e dados utilizados}

O estudo foi realizado na fazenda Cadeado, localizada em Lages, Santa Catarina, pertencente à empresa Klabin S/A (Figura 1). A fazenda tem área produtiva de 119,68 ha, divididos em quatro talhões com plantio de Pinus taeda com idade de 19 anos e espaçamento de 2,5 x 2,5 m. Em toda a área de plantio não foi aplicada nenhuma prática de desbaste durante todo o ciclo de vida da floresta.

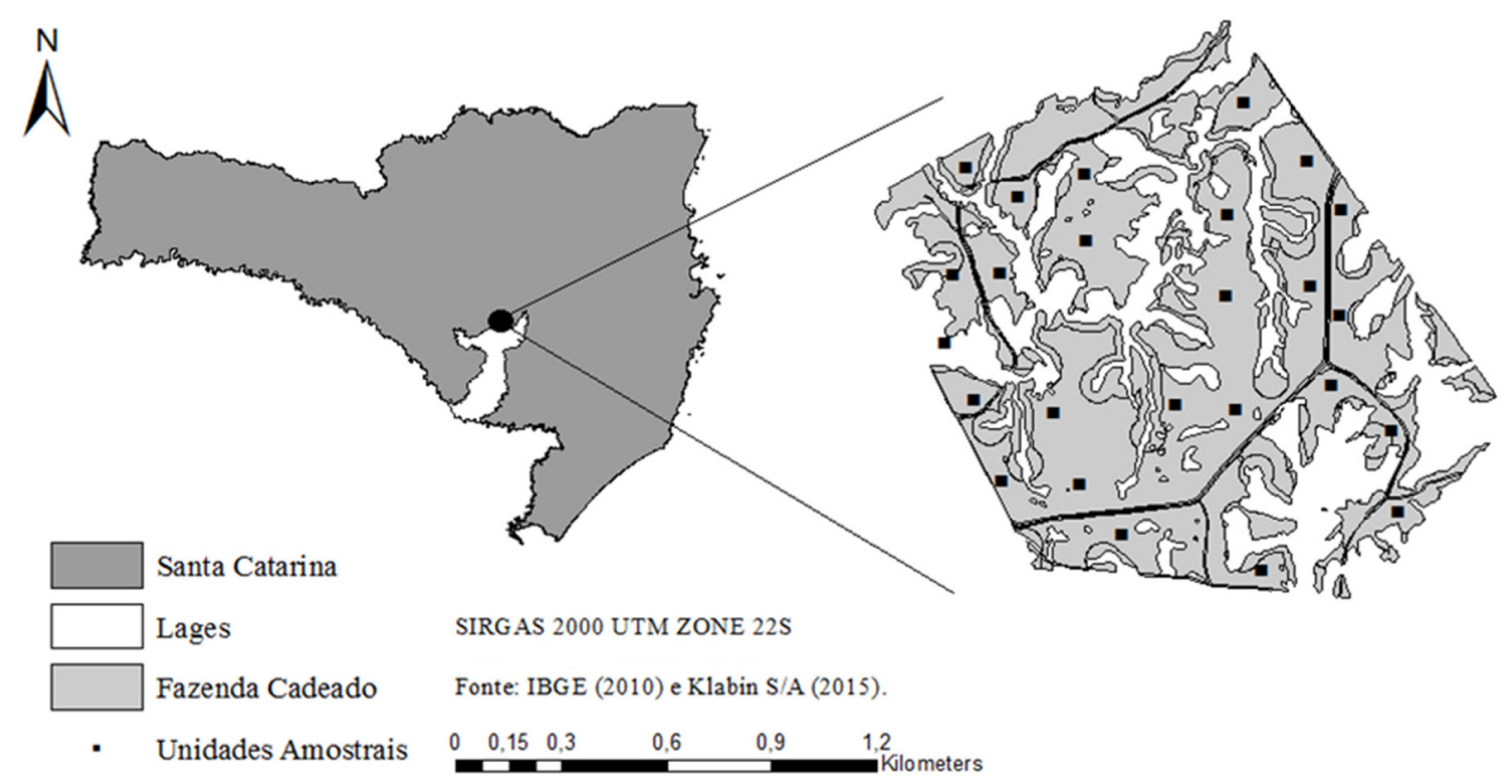

Figura 1. Mapa de localização da área de trabalho.

Figure 1. Location map of the research area. 
A área encontra-se em zona de ocorrência original da Mata Atlântica, na fitofisionomia Floresta Ombrófila Mista e os solos predominantes na região são Nitossolos Háplicos e Cambissolos Húmicos, sendo desenvolvidos a partir de rochas basálticas (EMBRAPA, 2006). A altitude média do local é de 902 m, o clima da região é classificado como Cfb, sendo subtropical do tipo mesotérmico úmido com verão ameno, segundo a classificação de Köppen. A precipitação total anual está entre 1300 e $1500 \mathrm{~mm}$ e a temperatura média anual é de $14,3^{\circ} \mathrm{C}$ e umidade relativa média do ar de $79,3 \%$ (PANDOLFO et al., 2002).

Os dados utilizados foram oriundos do inventário florestal realizado pela Klabin S/A no ano de 2015. Foram inventariadas e georreferenciadas 25 unidades amostrais de 25,6 x 24,1 m, sendo medidos os valores de circunferência à altura do peito (CAP) das árvores da unidade amostral e consideradas aquelas com CAP maior que $10 \mathrm{~cm}$. Nestas árvores foi medida a altura total utilizando hipsômetro. Posteriormente foram calculados o diâmetro à altura do peito (DAP) e a área basal para posteriormente obter valores de volume, e altura dominante.

\section{Estudo variográfico}

Primeiramente os dados de volume ( $\left.\mathrm{m}^{3} / \mathrm{ha}\right)$, área basal $\left(\mathrm{m}^{2} / \mathrm{ha}\right)$ e altura dominante $(\mathrm{m})$ foram submetidos à análise estatística descritiva e ao teste de Grubbs com $=5 \%$ para a detecção de possíveis valores discrepantes. O teste identifica valores discrepantes utilizando a Equação (1).

$$
Z=\frac{\left|x_{i}-\bar{x}\right|}{s}
$$

Em que: $Z$ é o valor crítico a ser testado; $\mathbf{x}_{\mathrm{i}}$ é uma observação da amostra $\mathbf{x}_{1}, \mathbf{x}_{2^{\prime}}, \ldots, \mathbf{x}_{\mathrm{n}^{\prime}} ; \bar{x}$ é a média amostral; S é o desvio padrão amostral.

Um valor é considerado discrepante, de acordo com o teste de Grubbs, quando Z é maior do que o valor crítico de referência correspondente.

Para testar a normalidade da distribuição dos dados foi aplicado o teste de Kolmogorov-Smirnov $\operatorname{com} \alpha=5 \%$. Este teste compara frequências acumulativas estimadas testando-as em relação à frequência acumulativa observada. Desta forma é possível avaliar, para cada valor proposto da variável em questão, qual o melhor ajuste, testando a hipótese relacionada à distribuição de probabilidade, no caso a distribuição normal (SCOLFORO; THIERSCHI, 1998).

Para cada característica dendrométrica foi calculado o semivariograma experimental omnidirecional utilizando o cálculo de diferentes classes de distâncias ou lags. Em seguida, para a análise da presença de anisotropia, quatro diferentes semivariogramas foram construídos nas direções $0^{\circ}$, $45^{\circ}, 90^{\circ}$ e $135^{\circ}$ relativas ao Norte, sendo comparados com o semivariograma omnidirecional. $\mathrm{O}$ semivariograma foi utilizado para mensurar a variabilidade espacial da variável regionalizada e para calcular os parâmetros de entrada da interpolação espacial por meio da krigagem.

Com a definição do semivariograma experimental foram ajustados os modelos teóricos dos tipos: exponencial (EXP), esférico (SPH) e gaussiano (GAU). Para o ajuste dos modelos foi utilizado o método de Máxima Verossimilhança (ML). A seleção do melhor modelo para cada característica dendrométrica foi realizada por meio do Critério de Informação de Akaike (AIC) (MELLO et al., 2005b). A seleção pelo AIC estabelece que o modelo com menor valor para um conjunto de dados é denominado de suporte (AICmin), e os demais são os modelos (i). Para a curva (i) diferenciar-se estatisticamente da curva suporte a expressão $\triangle \mathrm{AIC}=\mathrm{AICi}-\mathrm{AICmin}$ tem que ser maior ou igual a dois (BURNHAM; ANDERSON, 2002). Quando esta diferença não for encontrada opta-se pelo modelo com menor AIC (FARACO et al., 2008). Segundo Mello et al. (2005b), aplicando-se a condição de não-tendenciosidade, o valor populacional para o erro médio deve ser igual a zero e erro médio quadrático igual a um.

O grau de Dependência Espacial (DE\%) foi estabelecido por meio da relação entre a contribuição $(\mathrm{C} 1)$ e a variação estruturada $(\mathrm{C} 1+\mathrm{C} 0)$. Esta relação foi proposta por Biondi et al. (1994) em estudo do comportamento espacial do diâmetro do tronco e da área basal em floresta natural de Pinus ponderosa Douglas ex Lawson \& C. Lawson. A dependência espacial é fraca quando DE\% é menor que 25. É moderada quando DE\% está entre 25 e 75 e é forte quando DE\% é maior que 75.

As análises supracitadas no estudo variográfico foram realizadas no Software estatístico $R$ com o uso do pacote geoR (RIBEIRO JÚNIOR; DIGGLE, 2001). 


\section{Krigagem Ordinária (KO)}

Segundo (WEBSTER; OLIVER, 2000), a krigagem ordinária Ẑ(x) de variáveis regionalizadas $\mathrm{x}$ no ponto i é dada pela Equação (2).

$$
\hat{\mathrm{Z}}(x)=\sum_{i=1}^{n} \lambda i Z(x i)
$$

Em que: $\hat{Z}(\mathbf{x})$ é o valor estimado para o ponto não amostrado; $\lambda$ é o peso associado com o valor de $\mathbf{Z}(\mathbf{x i})$ no ponto amostrado $\mathbf{i}$.

A atribuição de pesos $(\lambda \mathrm{i})$ é realizada por meio da técnica dos multiplicadores de Lagrange que multiplica o inverso da matriz de semivariâncias entre os pontos amostrados pelo vetor de semivariâncias entre os pontos amostrados e o ponto estimado (PELISSARI et al., 2014). Esta técnica considera as diferentes distâncias no plano espacial, sendo que, cada unidade amostral observada contribui em distintos percentuais para as estimativas nos pontos não amostrados. Para a krigagem, que é um interpolador exato ou não enviesado, o somatório dos pesos deve ser obrigatoriamente igual a um $(\lambda \mathrm{i}=1)$.

\section{Ponderação pelo inverso da distância (IDW)}

O interpolador IDW assume a hipótese de que locais próximos são mais semelhantes entre si. Devido a isso, valores medidos próximos ao local terão maior influência na interpolação que outros distantes (KANEGAE JÚNIOR et al., 2006). O estimador IDW é dado pela Equação (3).

$$
\hat{\mathrm{Z}}(x)=\frac{\sum_{i=1}^{n} Z(x i) W i}{\sum_{i=1}^{n} W i}
$$

Em que: $\hat{Z}(x)$ é o valor estimado para o ponto não amostrado; Wi é o peso da i-ésima parcela amostral e Z(xi) valor observado da i-ésima parcela amostral.

O peso de um dado ponto amostral é calculado de acordo com a Equação (4) sendo que, para esse interpolador na determinação do peso apenas é considerado o inverso da distância euclidiana elevado no parâmetro da potência p que para este estudo foi utilizado igual a dois.

$$
W i=\frac{1}{d i^{p}}
$$

Em que: di é a distância entre cada ponto a ser estimado e observado e p o parâmetro da potência.

\section{Obtenção dos estratos}

Os métodos de estratificação testados neste estudo estão listados na Tabela 1. A obtenção dos estratos, por meio de ambos interpoladores KO e IDW, foi realizada com o Software ArcGis versão 10.1 (JOHNSTON, 2001) por meio da ferramenta Geostatistical Analyst.

\begin{tabular}{|c|c|c|}
\hline Método de estratificação & Método de Amostragem & Descrição \\
\hline Testemunha & Amostragem Casual Simples & $\begin{array}{l}\text { Adoção de toda a área como um estrato, ou seja, } \\
\text { sem nenhum controle da variância do volume. }\end{array}$ \\
\hline $\begin{array}{l}\text { Krigagem Ordinária do Volume } \\
\mathrm{em} \mathrm{m}^{3} . \mathrm{ha}^{-1}(\mathrm{KOV})\end{array}$ & Amostragem Casual Estratificada & $\begin{array}{l}\text { Estratos baseados no mapa de interpolação do } \\
\text { volume pela KO. }\end{array}$ \\
\hline $\begin{array}{l}\text { Krigagem Ordinária da Área } \\
\text { basal em } \mathrm{m}^{2} \text {.ha-1 }(\mathrm{KOG})\end{array}$ & Amostragem Casual Estratificada & $\begin{array}{l}\text { Estratos baseados no mapa de interpolação da } \\
\text { área basal pela KO. }\end{array}$ \\
\hline $\begin{array}{l}\text { Krigagem Ordinária da Altura } \\
\text { dominante em } \mathrm{m}(\mathrm{KOHd})\end{array}$ & Amostragem Casual Estratificada & $\begin{array}{l}\text { Estratos baseados no mapa de interpolação da } \\
\text { altura dominante pela KO. }\end{array}$ \\
\hline $\begin{array}{l}\text { IDW do Volume em } \mathrm{m}^{3} \cdot \text { ha }^{-1} \\
\text { (IDWV) }\end{array}$ & Amostragem Casual Estratificada & $\begin{array}{l}\text { Estratos baseados no mapa de interpolação do } \\
\text { volume pelo IDW. }\end{array}$ \\
\hline $\begin{array}{l}\text { IDW da Área basal em m².ha-1 } \\
\text { IDWG }\end{array}$ & Amostragem Casual Estratificada & $\begin{array}{l}\text { Estratos baseados no mapa de interpolação da } \\
\text { área basal pelo IDW. }\end{array}$ \\
\hline $\begin{array}{l}\text { IDW da Altura dominante em } \mathrm{m} \\
\text { (IDWHd) }\end{array}$ & Amostragem Casual Estratificada & $\begin{array}{l}\text { Estratos baseados no mapa de interpolação da } \\
\text { altura dominante pelo IDW. }\end{array}$ \\
\hline
\end{tabular}

Tabela 1. Caracterização dos métodos de estratificação e amostragem para processamento do inventário florestal. Table 1. Characterization of stratification and sampling methods used in the forest inventory processing.

Para a KO trabalhou-se em conjunto com os programas R e ArcGis, sendo que, o estudo variográfico, o qual define os parâmetros de entrada da $\mathrm{KO}$, foi realizado no $\mathrm{R}$, devido à possibilidade de ajustar modelos geoestatísticos pelo método Máxima Verossimilhança. Segundo Mello et al. 
(2005b) este método apresenta-se mais eficiente para dados simétricos quando comparado com o método dos Mínimos Quadrados Ordinários, utilizado pela ferramenta Geostatistical Analyst (JOHNSTON, 2001). Na interpolação pelo IDW utilizou-se apenas a ferramenta Geostatistical Analyst com $\mathrm{p}=2$. O número máximo de vizinhos utilizados foi igual a dez.

Optou-se por trabalhar com quatro estratos, devido à melhor operacionalidade (GUEDES et al., 2012; KANEGAE JÚNIOR et al., 2006); e a distribuição de classes dos dados dentro de cada estrato foi definida com o método de quebras naturais (Natural Breaks), também conhecido como Otimização de Jenks.

Após a interpolação de cada característica dendrométrica, por KO e por IDW, cruzou-se o mapa com a localização das parcelas com os diferentes mapas gerados pela interpolação. Assim foi possível visualizar a localização das parcelas dentro de cada estrato. A área dos estratos foi obtida por meio da multiplicação do valor da área de um pixel no terreno pela quantidade de pixels dentro de cada estrato.

\section{Processamento do inventário florestal}

Na Tabela 1 são apresentados os diferentes métodos utilizados no processamento do inventário florestal para avaliação dos métodos de estratificação.

A avaliação do melhor método de estratificação foi realizada por meio da comparação da variância $\left(S^{2}\right)$ e do erro de amostragem (ER\%) calculados para cada estratificador em relação à testemunha (ACS), já que os dois interpoladores não são comparáveis entre si devido à característica não enviesada da krigagem e inviesada do IDW (KANEGAE JÚNIOR et al., 2006). A eficiência do método de estratificação foi verificada avaliando-se a proporção de redução da variância do volume e do erro de amostragem quando comparados com a testemunha.

\section{RESULTADOS E DISCUSSÃO}

Os resultados da estatística descritiva para as diferentes características dendrométricas são apresentados na Tabela 2. Observa-se que o volume apresentou maior coeficiente de variação, seguido pela área basal e altura dominante, condição esperada dado que o volume estimado é obtido a partir da área basal e da altura das árvores. Essas informações são importantes para a primeira inferência sobre a produtividade na área. Por meio do teste Grubbs não foi constatada a presença de outliers na série de dados, resultando em melhores condições para o estudo variográfico (DIGGLE; RIBEIRO, 2007). Pelo teste Kolmogorov-Smirnov com valor crítico igual a 0,13 com 5\% de significância constatou-se que os dados são provenientes de uma população normal. Mesmo que a normalidade dos dados não seja uma exigência da geoestatística, esta condição possibilita o uso de propriedades estatísticas como a Máxima Verossimilhança, que é um importante método de ajuste de modelos geoestatísticos (DIGGLE; RIBEIRO, 2007).

Tabela 2. Análise estatística descritiva do volume, área basal e altura dominante no inventário florestal. Table 2. Descriptive statistical analysis of volume, basal area and dominant height on forest inventory.

\begin{tabular}{lccccccc}
\hline Atributo & Média & Mínimo & Máximo & Desvio Padrão & Coef. Variação (\%) & Teste Grubbs & Teste KS \\
\hline Vol $\left(m^{3} \cdot\right.$ ha $\left.^{-1}\right)$ & 649,28 & 551,00 & 748,00 & 49,29 & 7,59 & $2,00 S^{*}$ & $0,28 N S$ \\
G $\left(m^{2} \cdot h^{-1}\right)$ & 63,19 & 53,40 & 71,80 & 4,71 & 7,46 & $2,07 \mathrm{NS}^{*}$ & $0,73 \mathrm{NS}^{*}$ \\
$\mathrm{Hd}(\mathrm{m})$ & 24,51 & 23,10 & 25,90 & 0,58 & 2,36 & $2,42 \mathrm{NS}^{*}$ & $0,33 \mathrm{NS}$ \\
\hline
\end{tabular}

*NS: os dados seguem uma distribuição normal.

Na Tabela 3 estão apresentados os resultados do estudo variográfico. Não foi encontrado efeito anisotrópico em nenhuma das características dendrométricas analisadas (Volume, Área basal e Altura dominante) dessa forma, os modelos foram ajustados ao semivariograma omnidirecional.

Segundo a classificação proposta por Biondi et al. (1994), todas as características dendrométricas foram classificadas como moderada dependência espacial $(25<\mathrm{DE} \%>75)$.

O alcance (a) espacial médio do volume.ha ${ }^{-1}$ de 1.348,27 m sugere que as unidades amostrais do inventário florestal são consideradas correlacionadas espacialmente até esta distância. Este alcance é considerado longo, pois, a maior distância entre duas unidades amostrais na área de estudo é igual a $1.857 \mathrm{~m}$. 
Zech et al. - Uso de interpoladores espaciais na estratificação estatística de Pinus taeda

Tabela 3. Resultado do estudo variográfico para volume, área basal e altura dominante.

Table 3. Results for the variographic study for volume, basal area and dominant height.

\begin{tabular}{lllcccc}
\hline Atributo & Lag $(\mathbf{m})$ & Modelo & Efeito Pepita & Contribuição & Alcance $(\mathbf{m})$ & Dependência Espacial (\%) \\
\hline \multirow{2}{*}{ Vol $\left(\mathrm{m}^{3} \cdot \mathrm{ha}^{-1}\right)$} & 154,75 & Exp & $1.338,00$ & $2.271,00$ & $1.857,00$ & 62,92 \\
& 154,75 & Sph & $1.234,00$ & $1.070,00$ & $1.125,37$ & 46,44 \\
& 154,75 & Gau & $1.394,00$ & $1.003,00$ & $1.062,44$ & 41,84 \\
\hline \multirow{3}{*}{$\mathrm{G}\left(\mathrm{m}^{2} . \mathrm{ha}^{-1}\right)$} & 154,75 & Exp & 14,03 & 16,04 & 1857,00 & 53,34 \\
& 154,75 & Sph & 13,19 & 8,02 & 1131,16 & 37,81 \\
& 154,75 & Gau & 14,48 & 7,04 & 1021,64 & 32,71 \\
\hline \multirow{3}{*}{$\mathrm{Hd}(\mathrm{m})$} & 187,50 & Exp & 0,11 & 0,21 & 432,55 & 65,41 \\
& 187,50 & Sph & 0,16 & 0,16 & 462,00 & 50,64 \\
\hline
\end{tabular}

Elevados valores de efeito pepita (C0) podem ser explicados por estruturas com alcance menor do que a menor distância entre dois pontos (CHILES; DELFINER, 1999), ou seja, os dados apresentam elevada variação nas distâncias inferiores à amostrada. Exemplificando o modelo gaussiano para a variável volume, o efeito pepita corresponde a 58,16\% do patamar, ou seja, 41,84\% da variabilidade foi explicada pela correlação espacial. Esta análise é importante porque quanto maior esta proporção, piores serão as estimativas da krigagem, pois se assume que menor será a semelhança entre os vizinhos e a continuidade do fenômeno (VIEIRA, 1997). Para os modelos avaliados a menor proporção encontrada foi para o modelo exponencial para altura dominante $(35,00 \%)$ e o maior foi o modelo exponencial para área basal $(67,29 \%)$.

Da forma como foi conduzida a pesquisa, bem como a amostragem utilizada, não é possível afirmar que fatores estão influenciando este resultado. Acredita-se que a idade do povoamento elevou o efeito pepita, pois a competição entre as plantas afeta a relação espacial entre as unidades amostrais (KANEGAE JÚNIOR et al., 2007). Biondi et al. (1994), estudando um povoamento de Pinus ponderosa, também encontraram evidências de que, com o aumento da idade, o índice de dependência espacial diminui. Os autores afirmam que as limitações de crescimento impostas pela densidade das árvores não necessariamente geram taxas de crescimento dependentes da distância. Ressalta-se, que o efeito pepita e a continuidade espacial de características dendrométricas são altamente dependentes de um conjunto de fatores da estrutura da paisagem, não sendo possível atribuir o seu valor à somente um único fator (GILBERT; LOWELL, 1997); (GUNNARSSON et al., 1998); (KANEGAE JÚNIOR et al., 2007). Como solução, Pelissari et al. (2014) e Mello et al. (2005a) sugerem a aplicação de amostragens mais intensivas, que possibilitariam assim detectar mais detalhadamente uma continuidade espacial das características dendrométricas. A alocação de amostragens sistemáticas também pode auxiliar na solução do problema.

Como a condição para o uso da krigagem é que os dados tenham moderada a forte dependência espacial (YAMAMOTO; LANDIM, 2013), consideram-se os resultados encontrados como satisfatórios. Desta forma, a seleção do melhor modelo teórico ajustado ao conjunto de dados foi baseada no valor do AIC, que, quanto menor, melhor o modelo. Para a variável volume, o modelo selecionado foi o Gaussiano (AIC=268,3), já para a área basal e altura dominante foi o Esférico (AIC=152,4, AIC $=49,29$ respectivamente). Na Figura 2 estão apresentados os modelos selecionados ajustados ao semivariograma isotrópico.

Observa-se que os ajustes não foram bons, especialmente para a altura dominante.

Na Figura 3 são apresentados os mapas gerados a partir das diferentes interpolações (volume. ha $^{-1}$, área basal.ha' ${ }^{-1}$ e altura dominante).

Em termos espaciais pode-se afirmar que ambos interpoladores, quando comparados para mesma característica dendrométrica, definiram regiões semelhantes de produtividade, porém, variou a área em hectares de estrato para estrato. A alteração da proporção de cada estrato na área total implica em resultados diferentes do inventário florestal pelos meios de estratificação utilizados. Percebe-se que o método IDW superestimou a área das classes superiores. Resultados semelhantes foram encontrados por KANEGAE JÚNIOR et al. (2006) e Guedes et al. (2012) que atribui essa diferenciação a dois fatores: o primeiro é o fato do interpolador IDW ser viciado e a krigagem ser um interpolador exato; e o segundo é porque o IDW não considera a redundância de informações de parcelas próximas, diferentemente da krigagem, em que, na estimativa de pontos desconhecidos, o peso de parcelas agrupadas é muito pequeno. 

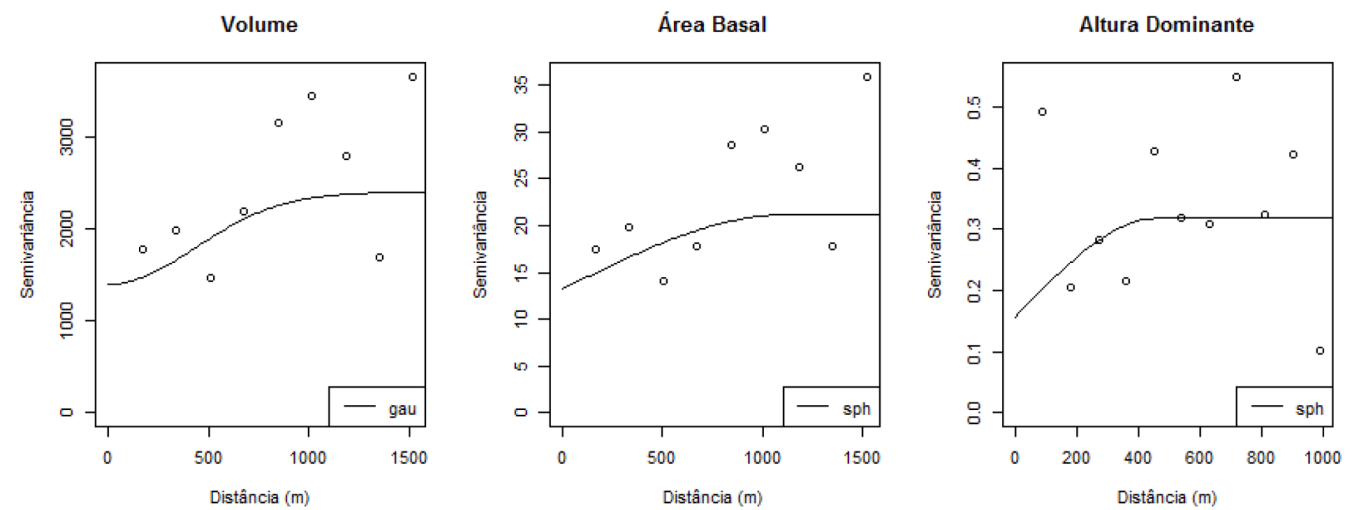

Figura 2. Semivariograma dos modelos selecionados. Linhas representam os modelos teóricos (gau=Gaussiano, $\mathrm{sph}=$ Esférico) e os pontos o semivariograma experimental.

Figure 2. Semivariogram for selected models. The lines represent the theoretic models (gau= Gaussian, $\mathrm{sph}=$ Spheric) and the points represent the experimental semivariogram.
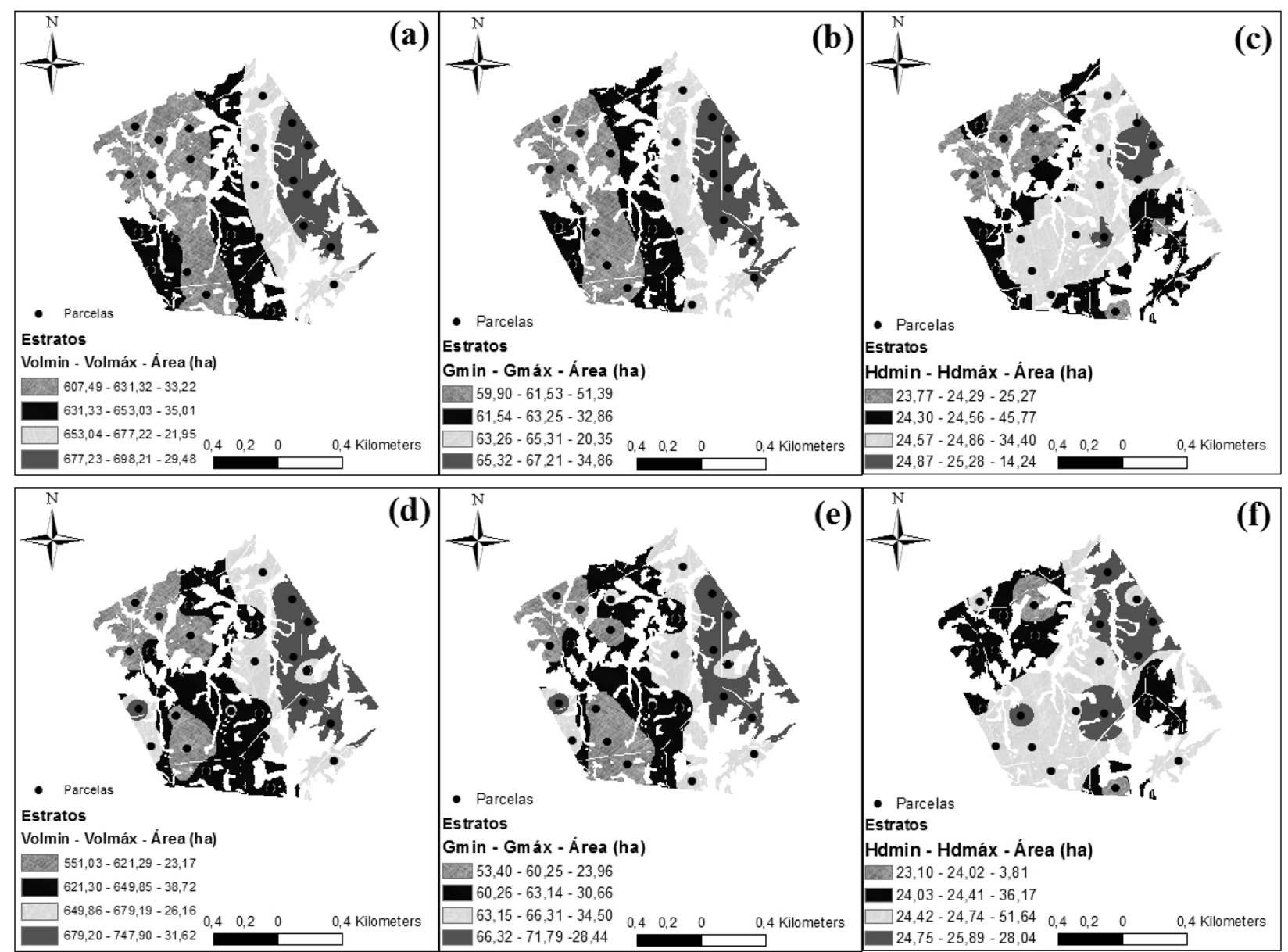

Figura 3. Mapas gerados por interpolação de: (a)volume/ha IDW, (b)área basal/ha IDW, (c)altura dominante IDW, (d)volume/ha KO, (e)área basal/ha KO, (f)altura dominante KO.

Figure 3. Maps generated form interpolation of: (a) volume/ha IDW, (b) basal area/ha IDW, (c) dominant height IDW, (d) volume/ha KO, (e) basal area/ha KO, (f) dominant height KO.

A variabilidade das características dendrométricas, como as apresentadas na Figura 3, contradiz a homogeneidade aparente em reflorestamentos, sendo correto afirmar que utilizar valores médios de unidades amostrais em áreas florestadas, não possibilita a correta caracterização da variabilidade das estruturas dendrométricas (PELISSARI et al., 2014). Dessa forma, combinar dados de inventários florestais com análises geoestatísticas permite produzir mapas que fornecem a melhor espacialização de características dendrométricas para dada área. Para Pelissari et al. (2014) a utilização de um mapa desse tipo em empreendimentos florestais otimiza a aplicação de tratamentos silviculturais, como, por exemplo, possibilita realizar desbastes somente em locais que apresentam elevados valores de área basal.ha-1 ${ }^{-1}$ 
Os resultados do volume médio, variância, erro absoluto e erro relativo calculados no inventário utilizando estimadores da ACS e ACE estão apresentados na Tabela 4.

Tabela 4. Estimadores considerando a Amostragem Casual Simples (ACS) e Amostragem Casual Estratificada (ACE) com estratos obtidos por meio da KRG e IDW.

Table 4. Simple Casual Sampling and Stratified Casual Sampling estimators obtained through KRG and IDW.

\begin{tabular}{|c|c|c|c|c|}
\hline Estimadores & Média $\left(\mathrm{m}^{3} \cdot \mathrm{ha}^{-1}\right)$ & Variância $S^{2}\left(m^{2} \cdot \text { ha }^{-1}\right)^{2}$ & Erro Absoluto E.A.(m $\left.{ }^{3} \cdot \mathrm{ha}^{-1}\right)$ & Erro Relativo E.R.(\%) \\
\hline ACS & 649,28 & 2429,04 & 20,21 & 3,11 \\
\hline ACE - KOV & 653,84 & 1036,99 & 14,58 & 2,23 \\
\hline ACE - KOG & 656,37 & 945,50 & 14,43 & 2,20 \\
\hline $\mathrm{ACE}-\mathrm{KOHd}$ & 653,63 & 1766,65 & 18,74 & 2,87 \\
\hline ACE - IDWV & 649,33 & 504,87 & 9,25 & 1,43 \\
\hline ACE - IDWG & 649,35 & 609,77 & 10,16 & 1,56 \\
\hline ACE - IDWHd & 648,74 & 2659,91 & 22,41 & 3,45 \\
\hline
\end{tabular}

Os melhores estratificadores do método KO foram aqueles baseados na área basal e no volume, sendo que o método KOG reduziu 73,42\% da variabilidade do volume e o KOV 57,30\%. Com relação ao erro de amostragem os métodos KOG e KOV, comparados com a ACS, reduziram 28,59\% e $27,85 \%$, respectivamente. Pelo método IDW os resultados foram semelhantes e os melhores estratificadores foram aqueles baseados no volume e área basal, na qual reduziram a variabilidade do volume em $79,21 \%$ e $74,89 \%$ e o erro de amostragem em $54,23 \%$ e $48,72 \%$, respectivamente.

Por outro lado, o método de pós-estratificação menos eficiente foi aquele baseado na altura dominante para os dois interpoladores. O método KOHd reduziu a variabilidade do volume e o erro de amostragem em 27,26\% e 7,27\%. O método IDWHd aumentou a variabilidade do volume e o erro de amostragem em 9,50\% e 10,88\%.

Observa-se que o interpolador IDW apresentou elevada eficiência para volume e área basal, assim como encontrado por Guedes et al. (2012) e KANEGAE JÚNIOR et al. (2006). Segundo os mesmos autores, esta eficiência é devida à característica determinística deste interpolador, ou seja, gera estimativa a partir de pontos medidos e se baseiam apenas na configuração espacial da amostra. Isso o torna eficiente apenas para a amostragem em estudo, não garantindo que a eficiência seja mantida se existirem inúmeras amostragens sobre o mesmo projeto. Em situações onde o grau de continuidade espacial é fraco ou o analista não saiba detectar corretamente a dependência espacial, o uso do interpolador IDW na pós-estratificação é uma alternativa interessante, pois, mesmo sendo viciado em comparação com a krigagem, apresenta aumento da precisão nas estimativas (KANEGAE JÚNIOR et al., 2006); (ASSIS et al., 2009); (GUEDES et al., 2012).

Para a KO, KANEGAE JÚNIOR et al. (2006) encontraram reduções na variabilidade do volume quando comparados ACS e ACE, sendo que o uso do mapa de volume/ha na pós-estratificação de povoamentos jovens de Eucalyptus sp., promoveu uma redução da variabilidade da área não estratificada igual a $47 \%$ e, consequentemente, 32\% do erro de amostragem. Guedes et al. (2012) também relataram que o uso do mapa de volume/ha, em povoamentos de Eucalyptus sp. com moderada e forte dependência espacial, reduz em média 47\% e 65,7\% o erro de amostragem, respectivamente. Assis et al. (2009) e Alvarenga et al. (2012) também encontraram redução do erro de amostragem com o uso de mapas gerados pela interpolação do volume.ha ${ }^{-1}$ pela KO.

Portanto, com base nos resultados apresentados, é possível afirmar que os interpoladores espaciais, KO e IDW, são ferramentas eficientes na estratificação florestal, por meio dos quais, consegue-se reduzir a variabilidade da característica de interesse e consequentemente o erro de amostragem. Este resultado possibilita que, em futuros inventários, possa-se utilizar a menor intensidade de amostragem com a mesma precisão requerida. Isto implica diretamente na redução dos custos, uma vez que estes estão alicerçados ao número de unidades amostrais e à precisão desejada (CESARO et al., 1994; GUEDES et al., 2012; KANEAGAE JÚNIOR et al., 2006; SANQUETTA et al., 2006).

\section{CONCLUSÕES}

As características dendrométricas analisadas: Volume, Área basal e Altura dominante, apresentaram moderada dependência espacial. Além disso, ambos os interpoladores analisados se mostraram eficientes na estratificação florestal de povoamentos de Pinus taeda em final de rotação. 
As estratificações produzidas com os parâmetros área basal e volume apresentaram melhores resultados que aquela produzida com altura dominante.

A amostragem casual estratificada com interpoladores espaciais se mostrou vantajosa em relação à amostragem casual simples, apresentando-se como uma estratégia a ser considerada para redução da variabilidade em inventários florestais.

\section{REFERÊNCIAS BIBLIOGRÁFICAS}

ALVARENGA, L. H. V.; MELLO, J. M.; GUEDES, I. C. L.; SCOLFORO, J. R. S. Desempenho da estratificação em um fragmento de cerrado stricto sensu utilizando interpolador geoestatístico. Cerne, Lavras, v. 18, n. 4, p. $675-681,2012$.

ASSIS, A. L.; MELLO, J. M.; GUEDES, I. C. L.; SCOLFORO, J. R. S.; OLIVEIRAS, A. D. Development of a sampling strategy for young stands of Eucalyptus sp. using geostatistics. Cerne, Lavras, v. 15, n. 2, p. 166-173, 2009.

BIONDI, F.; MYERS, D. E.; AVERY, C. C. Geostatistically modeling stem size and increment in an old-growth forest. Canadian Journal of Forest Research, Ottawa, v. 24, n. 7, p. 1354-1368, 1994.

BURNHAM, K.P.; ANDERSON, D.R. Model selection and multimodel inference. 2.ed. New York: Springer, 2002. $350 \mathrm{p}$.

CESARO, A.; ENGEL, O. A.; FINGER, C. A. G. Comparação dos métodos de amostragem de área fixa, relascopia e de seis árvores, quanto a eficiência, no inventário florestal de um povoamento de Pinus sp, Ciência Florestal, Santa Maria, v. 4, n. 1, p. 97-108, 1994.

CHILES, J. P.; DELFINER, P. Geostatistics: modeling spatial uncertainty. New York: Wiley, 1999.

DIGGLE, P. J.; RIBEIRO JÚNIOR, P. J. Model based geoestatistics. Londres.: Springer, 2007.

EMBRAPA. Sistema Brasileiro de Classificação de Solos. Rio de Janeiro: Embrapa Solos, 2006.

FARACO M. A.; URIBE-OPAZO, M. A.; SILVA, E. A.; JOHANN J. A.; BORSSOI, J. A. Seleção de modelos de variabilidade espacial para elaboração de mapas temáticos de atributos físicos do solo e produtividade da soja. Revista Brasileira de Ciência do Solo, Viçosa, v. 32, n. 2, p. 463-476, 2008.

GILBERT, B.; LOWELL, K. Forest attributes and spatial autocorrelation and interpolation: effects of alternative sampling schemata in the boreal forest. Landscape and Urban Planning, Amsterdam, v. 37, n. 3-4, p. 235244p., 1997.

GUEDES, I. C. L.; MELLO, J. M.; OLIVEIRA, A. D.; SILVA, S. T.; SILVA, S. T.; SCOlFORO, J. R. S. Técnicas geoestatísticas e interpoladores espaciais na estratificação de povoamentos de Eucalyptus sp. Ciência Florestal, Santa Maria, v. 22, n. 3, p. 541-550, 27 set. 2012.

GUNNARSSON, F.; HOLM, S.; HOLMGREN, P; THURESSON, T. On the potential of kriging for forest management planning. Journal of Forest Research, Oslo, v. 13, n. 1-4, p. 237-245, 1998.

JOHNSTON, K. Using ArcGIS Geostatistical Analyst. Redlands: Environmental Systems Research Institute ESRI, 2001. 306 p.

KANEGAE JÚNIOR JÚNIOR, H.; SCOLFORO, J. R.; MELLO, J. M.; OLIVEIRA, A. D. Avaliação de interpoladores estatísticos e determinísticos como instrumento de estratificação de povoamentos clonais de Eucalyptus sp. Cerne, Lavras, v. 12, n. 2, p. 123-136, 2006.

Sci. For., Piracicaba, v. 46, n. 117, p. 87-96, mar. 2018 DOI: dx.doi.org/10.18671/scifor.v46n117.08 
Zech et al. - Uso de interpoladores espaciais na estratificação estatística de Pinus taeda

KANEGAE JÚNIOR, JÚNIOR, H.; MELLO, J. M.; SCOLFOTO, J. R.; OLIVEIRA, A. D. Avaliação da continuidade espacial de características dendrometricas em diferentes 70 idades de povoamentos clonais de Eucalyptus sp. Revista Árvore, Viçosa, v. 31, n. 5, p. 859-866, 2007.

LUNDGREN, W. J. C.; SILVA, J. A. A.; FERREIRA, R. L. C. Estimação do volume de madeira de eucalipto por cokrigagem, krigagem e regressão. Cerne, Lavras, v. 21, n. 2, p. 243-250, 2015.

MELLO, J. M.; SCOLFORO, J. R. S. Análise comparativa de procedimentos de amostragem em um remanescente de Floresta Estacional Semidecídua Montana. Revista Árvore, Viçosa, v. 24, n. 1, p. 55-62, 2000.

MELLO, J. M.; BATISTA, J. L.; OLIVEIRA, M. S.; RIBEIRO JÚNIOR, P. J. Estudo da dependência espacial de características dendrométricas para Eucalyptus grandis. Cerne, Lavras, v. 11, n. 2, p. 113-126, 2005 a.

MELLO, J. M.; BATISTA, J. L. F.; TIBEIRO JÚNIOR, P. J. R.; OLIVEIRA, M. S. Ajuste e seleção de modelos espaciais de semivariograma visando à estimativa volumétrica de Eucalyptus grandis. Scientia Forestalis, Piracicaba, n. 69, p. 25-37, 2005b.

PANDOLFO, C. Atlas climatológico do Estado de Santa Catarina. Florianópolis: Epagri, 2002.

PELISSARI, A. L.; FIGUEIREDO FILHO, A.; CALDEIRA, S. F.; MACHADO, S. A. Geoestatística aplicada ao manejo de povoamentos florestais de teca, em períodos pré-desbaste seletivo, no estado do Mato Grosso. Revista Brasileira de Biometria, Lavras, v. 32, n. 3, p. 430-444, 2014.

PÉLLICO NETTO, S.; BRENA, D. A. Inventário Florestal. Curitiba: Edição dos autores, 1997.

RIBEIRO JÚNIOR, P. J.; DIGGLE, P. J. geoR: a package for geostatistical analysis. R news, v. 1, n. 2, p. 14-18, 2001.

SANQUETTA, C. et al. Inventários Florestais: Planejamento e Execução. Curitiba: Multi-Graf, 2006. 270 p.

SCOLFORO, J.R.S., THIERSCHI, A. Estimativas e testes da distribuição de frequência diâmétrica para Eucalyptus camaldulensis, através da distribuição Sb, por diferentes métodos de ajuste. Scientia Forestalis, Piracicaba, v. 54, p. 93-106, 1998.

VIEIRA, S. R. Variabilidade espacial de argila, silte e atributos químicos em uma parcela experimental de um latossolo roxo de Campinas (SP). Bragantia, Campinas, v. 56, n. 1, p. 181-190, 1997.

WEBSTER, R.; OLIVER, M. A. Geostatistics for environmental scientists. West Sussex: John Wiley \& Sons, 2000 .

YAMAMOTO, J. K.; LANDIM, P. M. B. Geoestatística: conceitos e aplicações. São Paulo: Oficina de Textos, 2013.

Recebido em 11/02/2017

Aceito em 21/08/2017 